\title{
Position Estimation with Fuzzy Logic Based Dead Reckoning for GPS-Denied Environment
}

\begin{tabular}{|c|c|c|}
\hline \multicolumn{3}{|c|}{ Murat LÜY $^{1}$, Faruk ULAMIŞ$^{2}$, Ertuğrul ÇAM $^{1}$} \\
\hline $\begin{array}{c}{ }^{1} \text { Kırlkkale Üniversitesi, } 1 \\
{ }^{2} \text { Kirlkkale Universit }\end{array}$ & $\begin{array}{l}\text { dislik Fakültesi Elektrik Elektror } \\
\text { ıcllar Hüseyin Aytemiz Meslek Y }\end{array}$ & $\begin{array}{l}\text { hendisliği, KIRIKKALE, TURKEY } \\
\text { Dkulu, KIRIKKALE, TURKEY }\end{array}$ \\
\hline Başvuru/Received: 06/05/2017 & Kabul/Accepted: 06/09/2017 & Son Versiyon/Final Version: 26/12/2017 \\
\hline
\end{tabular}

\begin{abstract}
The importance of security precautions has been increased as a consequence of raised terrorist incidents. Terrorist acts that move into the city from country sides have made tracking of security and military personnel more important. GPS signals, densely utilized for this tracking, pose a problem in signal blockage situation and GPS-denied environments. The main goal of this research is that to develop a practical positioning system for walking military personnel in GPS denied environments. The system is very useful for GPS signal blockage environments, such as tunnels, thick-walled structures and dense high rise buildings, etc.

Proposed system contains a 6-degree of freedom (DOF) inertial measurement unit (IMU) and digital compass attached to the military personnel's shoe. Tri-axis accelerometer and gyroscope measurements are obtained by IMU. These measurements are applied to fuzzy logic algorithms and total displacement for each step is calculated. Digital compass is utilized to determine direction of personnel. Thus, current location has been estimated with the known starting point.
\end{abstract}

\section{Key Words}

"Inertial Measurement Unit, Fuzzy Logic, GPS-denied environment, Dead reckoning." 


\section{INTRODUCTION}

Nowadays, navigation is a crucial part of our daily life. However, position estimation for outdoor can be obtained by GPS easily, positioning indoor environment is still a challenging research field which has various application like emergency first responder, military events and security personals (Riebeck et al., 2007; Foxlin, 2005; Samama et al., 2008)

Although there are many studies on positioning indoor environment, it is still open area to research. Indoor positioning system can be separated two branch broadly. First one is infrastructure based system which are Wi-Fi (Retscher et al., 2006), Ultra-Wide Band (UWB) (Ingran et al., 2004), Radio Frequency identification (RFID)(Huang et al., 2015). Infrastructure based indoor positioning requires sensors or beacons that must be placed indoor environment beforehand.

Wi-Fi, RFID and UWB methods are based on radio signals. So the most significant problem in these methods is signal blockage problem. In particular, although UWB offers high precision solutions for indoor positioning, there is a need for infrastructure setup in these three methods and IR methods. So the infrastructure means cost. But besides the problem of cost; In case of an emergency such as an earthquake, fire, or in the event of a power failure, these infrastructures may become damaged or become unusable. As a result, systems that require infrastructure are problematic, especially in military and emergency situations.

The visual based approach is an indoor positioning method that does not require infrastructure setup. In recent years, the fact that visual sensors are cheap and available on all smartphones has brought this method to the forefront. So visual-based approach has become popular for indoor positioning especially robotic navigation and unmanned aerial vehicles (JongBae \& HeeSung, 2008). However, there are some disadvantages using visual based approach in practice. The first one is a huge database must be available before navigation. The second disadvantage is the need for high processing power when comparing images with thousands of images in the database. On the other hand in situations such as blurred images and low ambient light, the system cannot perform image matching.

Recent developments in micro-electromechanical systems (MEMS) and fiber optic gyroscope technologies have reduced the size of inertial sensors and made them cheaper. On the other hand, as a result of these sensors produce their own data, there is not problems like signal blockage or signal jamming.

There are several methods for inertial sensor based indoor positioning (Widyawan et al., 2008), (Ojeda\&Borenstein, 2008). One of the most powerful and easy of these methods is pedestrian dead reckoning (Jimenez et al., 2009). The main idea of pedestrian dead reckoning method is to utilize accelerometer signals to detect steps, estimate step length and propagate position using a measured heading (such mechanization requires that possible means of transportation other than walking are excluded). Heading can be computed using gyro measurements, digital compass or using both.

The main goal of the research presented here is to develop an algorithm for Pedestrian Dead Reckoning (PDR) based indoor position system. For this purpose, a foot mounted system which contains 6-degree of freedom (DOF) inertial measurement unit (IMU), a magnetic compass and a microprocessor is utilized for indoor PDR system. The main part of PDR system is to determine step length and it is quite depended on walking status. For determining walking status, a fuzzy logic walking status (FLWS) algorithm is proposed in this study.

\section{SYSTEM ARCHITECTURE}

The algorithm for achieving an accurate positioning solution is represented as shown in Fig. 1. Hardware of the system includes three components called MPU 6050 Inertial Measurement Unit (IMU), HMC 5883 Digital Compass and Arduino Nano as a microprocessor. MPU6050 IMU has 6-Degree of freedom (DoF) and generates 3-axis accelerometer and gyroscope measurements. HMC 5883 digital compass has 12 -bit ADC to enable $1^{\circ}$ to $2^{\circ}$ compass heading accuracy for determining heading. IMU and digital compass have I2C serial bus for allowing easy interface with Arduino platform.

Each sensor is utilized for PDR algorithm. PDR has three main step to estimate position. The first one is step detection, second one is heading estimation. The last and the most important one is step length estimation. IMU is utilized to detect each step. After step detection, walking status of pedestrian (slow, fast and running) is recognized by Fuzzy Logic Walking Status (FLWS) algorithm. Then step length is estimated by PDR using number of step and walking status of each step. Digital compass is used to determine heading of pedestrian. 


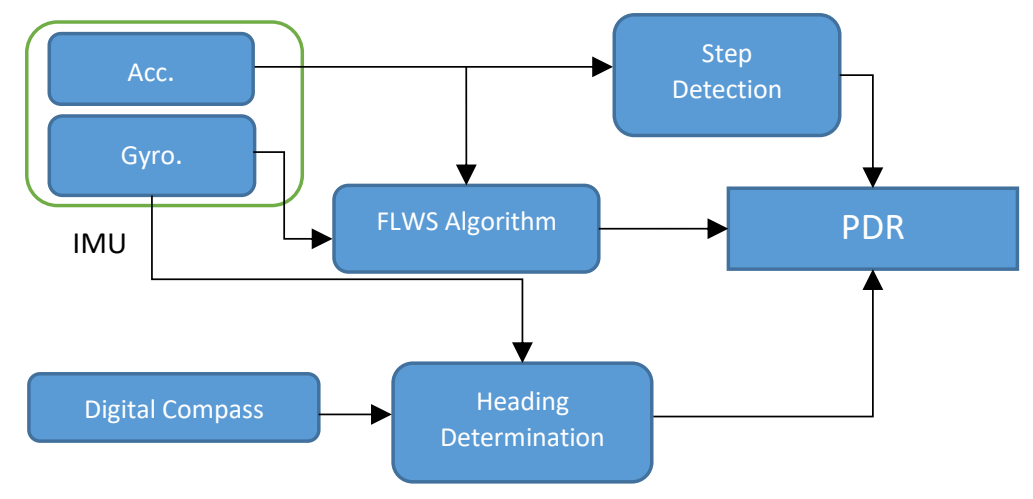

Fig. 1. FLWS Based PDR Algorithm

\section{PDR SYSTEM}

The basic pattern of pedestrian motion during a walk is a repeatable and cyclical event. Each step consist of two phase as shown figure 2. The first one called "stand phase" is a duration while foot is on the ground and second one called "swing phase" which is start with foot's lifting off the ground and carried forward to begin the stance phase again.

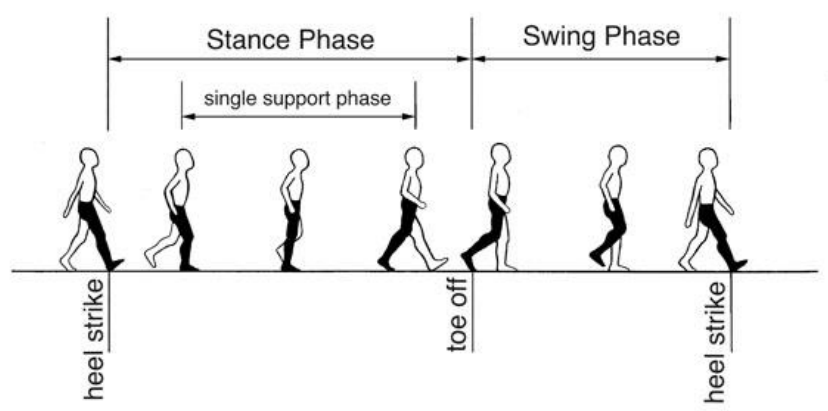

Fig. 2. Swing and stance phases while walking

PDR, which is a method adding distance travelled to the known start point, is proper solution to estimate position of pedestrian for indoor environment. To accomplish an accurate estimation, PDR has three main parts: step detection, heading estimation and step length estimation. After these three module, PDR algorithm calculates the total displacement.

\subsection{Step Detection}

The first step of the proposed algorithm is step detection. In order to get reliable estimation, step detection must be done accurately. When the location of sensor position is considered, many type of studies have already been done, e.g. the waist, trunk, leg, foot or even the head (Huang et al., 2015). In this study, foot-mounted IMU location have been implemented because of its two advantages. The first advantage of foot-mounted system is to easy detection of Zero Velocity Update (ZUPT) and the second one is make step detection easy compared to other IMU locations. Tri-axis accelerometer and tri-axis gyroscope measurements can be obtained as shown figure 3. 

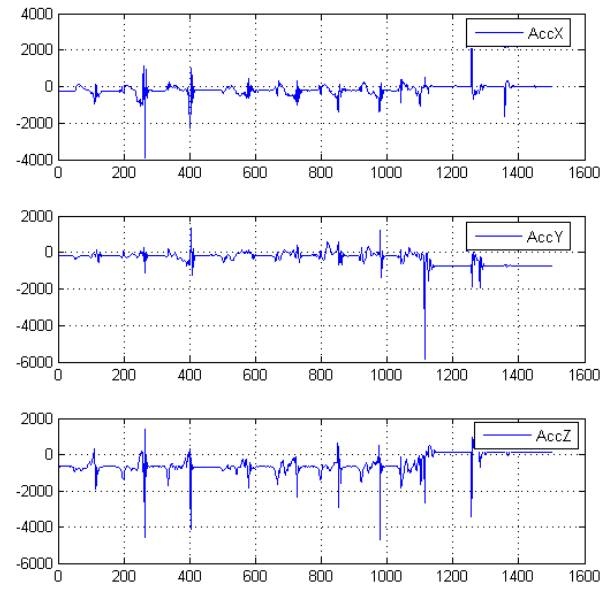
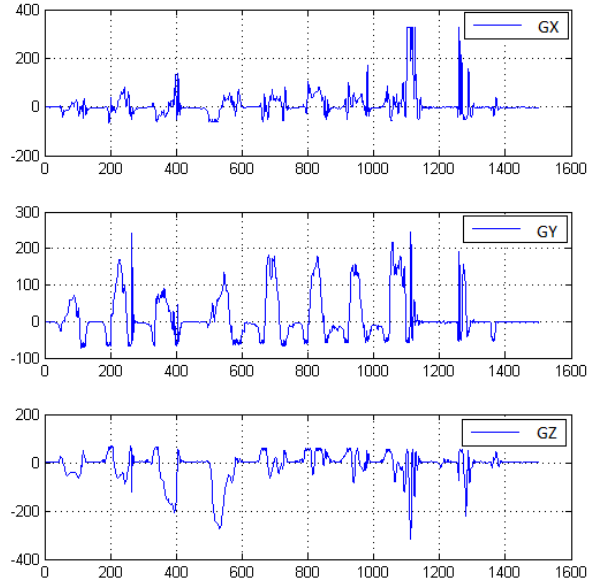

Fig. 3. Tri-axis gyroscope and accelerometer measurement from foot-mounted IMU

There are many techniques for step detection in the literature (acceleration peaks, magnitudes and thresholding). Most of this techniques use the data obtained by accelerometer and gyroscope. In this study, acceleration signal is analyzed to detect step. There are two methods which is used acceleration signals; peak detection and zero-crossing detection.

Zero crossing method counts zero velocity level to determine each step. For minimizing or eliminating false step detection, a time interval thresholding has been utilized. The disadvantage of this method is that the time interval between footfalls varied for subjects. As a consequence, it is very hard to detect step accurately without calibration process using this method.

Second method is to detect accelerometer measurements peak values. The peaks of acceleration values correspond to the step occurrences. Because the peaks of acceleration is generated by vertical impact when the foot hits the ground. Peaks of acceleration for step detection is used in this study.

\subsection{Heading Estimation}

Broadly, heading of pedestrian can be done with three methods; gyroscope signal, digital compass and both sensors. IMU gives tri-axis angular velocity and heading is obtained by integration of this signal. While integration, not only true values but also noise and errors like drift are processed. As a result, errors are accumulated over time. So, gyroscope measurement cannot used alone generally. On the other hand; digital compass uses earth's magnetic fields and this magnetic field is relatively weak and electrical fields can distort the output of this kind of sensors. The characteristics of two sensors are summarized in Table 3, where advantage of one sensor is disadvantage of the other (Kim et al., 2004).

Table 1. Comparision magnetic compass and gyroscope based system

\begin{tabular}{|c|c|c|}
\hline & Advantage & Disadvantage \\
\hline $\begin{array}{c}\text { Magnetic } \\
\text { compass }\end{array}$ & $\begin{array}{c}\text { Absolute azimuth } \\
\text { Long term stable accuracy }\end{array}$ & $\begin{array}{c}\text { Unpredictable external } \\
\text { disturbances }\end{array}$ \\
\hline Gyroscope & $\begin{array}{c}\text { No external disturbances } \\
\text { Short term accuracy }\end{array}$ & Relative azimuth drift \\
& Sherathe \\
\hline
\end{tabular}

To overcome two kind of disadvantages; both sensors are used generally. Gyroscope is used for correcting the magnetic disturbance, at the same time magnetic compass is used for determining and compensating the errors occurred by inertial sensor.

\subsection{Step Length Estimation}

Total displacement is calculated by estimating step length in every valid detected step. Generally, estimation of step length can be separated into two methods: static method and dynamic method. In static method, every step length are assumed to same, which can be determined through equation (1).

step_size = height $x k$

with $\mathrm{k}$ equal to 0.415 for men and 0.413 for women. 
Contrary to static method, every step length are not equal. Especially, walking status of pedestrian is the most effective factor for step length. In this study, walking status of pedestrian is recognized by a fuzzy logic algorithm. After recognizing walking status of pedestrian, step length can be estimated more accurately.

The main idea of PDR is walking distance and heading of the walking period to current position of pedestrian from a known previous position which can be seen Figure 4. (Judd \& Levi, 1996)

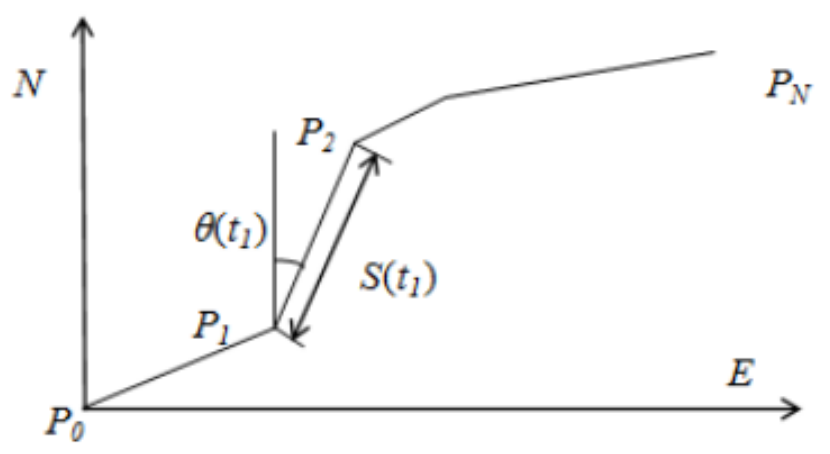

Fig. 4. Calculation of step length for each stride.

Total displacement can be calculated 1 and 2 .

$E\left(t_{n}\right)=E\left(t_{0}\right)+\sum_{0}^{n-1} S(t i) x \sin (\theta(t i))$

$N\left(t_{n}\right)=N\left(t_{0}\right)+\sum_{0}^{n-1} S(t i) x \cos (\Theta(t i))$

Where $\mathrm{E}(\mathrm{t} 1), \mathrm{N}(\mathrm{t} 1)$ is previous position, $\mathrm{E}(\mathrm{tn}), \mathrm{N}(\mathrm{tn}))$ is current position and $\theta$ is heading during the walking period.

For the length of each step can be calculated as follow;

$S(t)=C_{\text {walking status }} x N S$

Where $\mathrm{C}$ is coefficient of walking status and NS is number of steps taken in the same direction

\subsection{Fuzzy Logic Walking Status Algorithm}

The Fuzzy logic concept is first introduced by L. A. Zadeh in 1965 [13]. Since it was introduced, it has been used for solving many kind of complex problem related to human experience. Basically, Fuzzy logic seems closer to the way our brains work and it solves problems by considering all available information and making the best possible decision given the input, in the same way that humans do.

Step length estimation for each stride is crucial and the most effective parts of the PDR system considerably. Since the total displacement is estimated by sum of all steps, total error grows exponentially. In this study, for minimizing the errors for each step a fuzzy logic walking status (FLWS) algorithm is proposed shown figure 5.

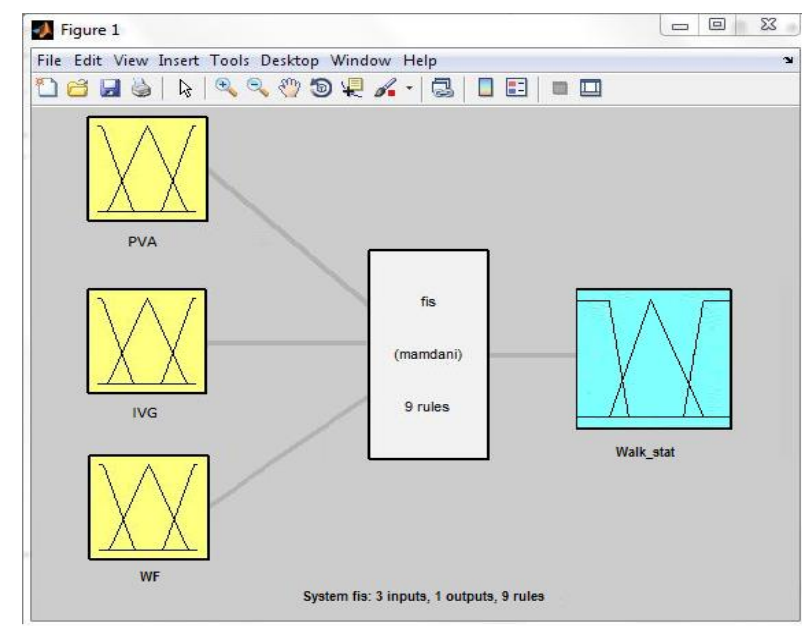

Fig. 5. Proposed fuzzy logic algorithm 
There are three main factors affecting step length is that; stride related on walking frequency (WF), peak value of accelerometer (PVA) and integration value of gyroscope (IVG). These measurements are input for the proposed fuzzy logic algorithm. The output of the algorithm is walking status of pedestrian.

Status of walking which are "stop, normal walking and running" is determined by FLWS algorithm. The output of FLWS algorithm is shown figure 6.

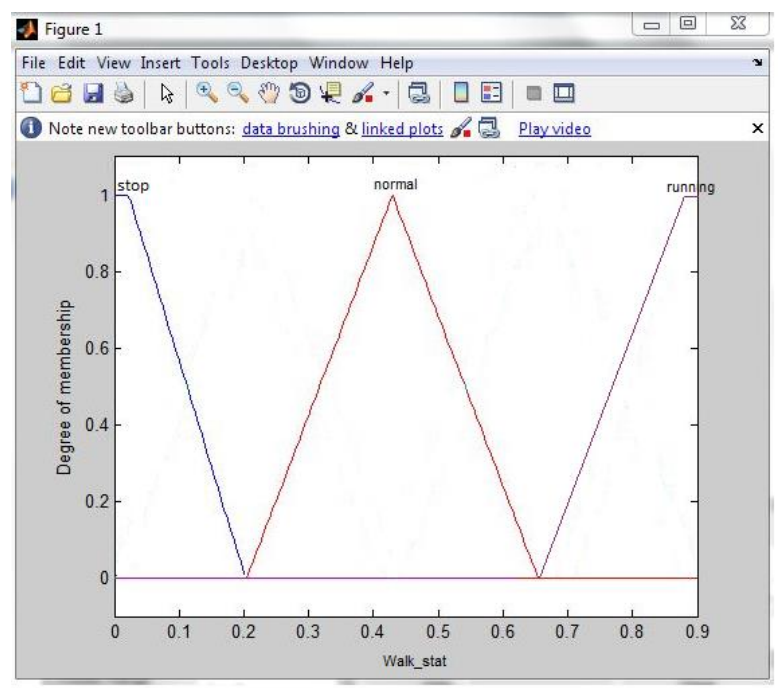

Fig. 6. Output of fuzzy algorithm.

\section{EXPERIMENTAL RESULTS}

In order to verify proposed PDR algorithm, various experiments were conducted. Thick black shown figure 7 is the route of a pedestrian. The pedestrian was started at the green point and followed the route and finished at the red point. Before perform PDR algorithm, 45 experiments were done to determine walking status and the result can be seen table 2 .

Table 2. Result of FLWS algorithm

\begin{tabular}{|l|c|c|c|c|}
\hline & $\begin{array}{l}\text { Number of } \\
\text { experiment }\end{array}$ & True & False & percentage \\
\hline Stop & 5 & 5 & 0 & $100 \%$ \\
\hline Normal walking & 20 & 19 & 1 & $95 \%$ \\
\hline Running & 20 & 19 & 1 & $95 \%$ \\
\hline
\end{tabular}

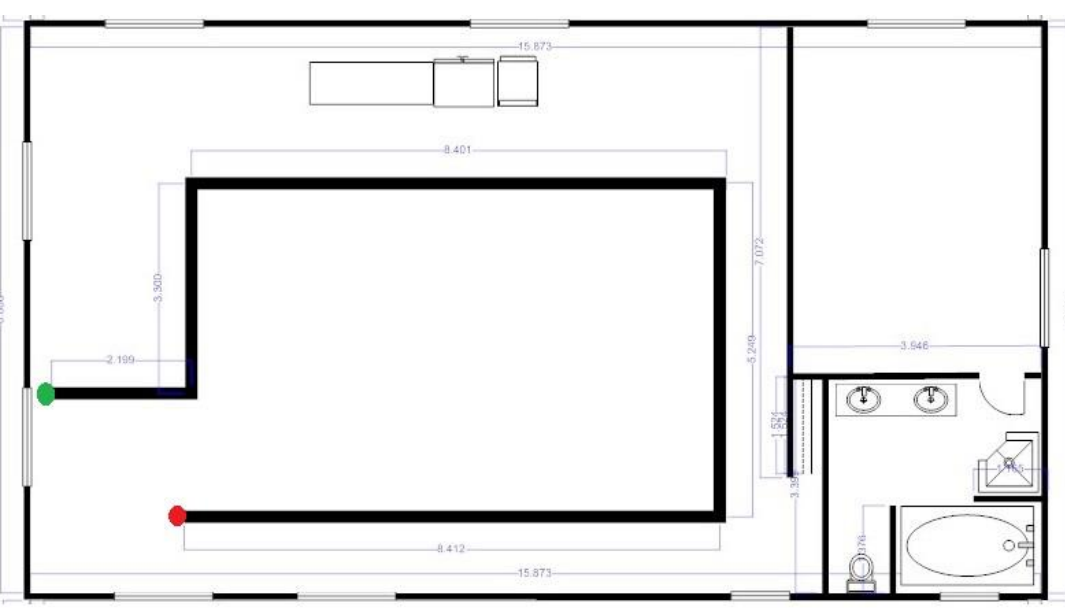

Fig. 7. Route of Pedestrian 
The first experiment was performed at normal walking speed. Figure 8 shows the total displacement of pedestrian. Total displacement error is $3 \%$ and second experiment was performed at running which result can be seen figure 9 and total displacement error is $7 \%$.

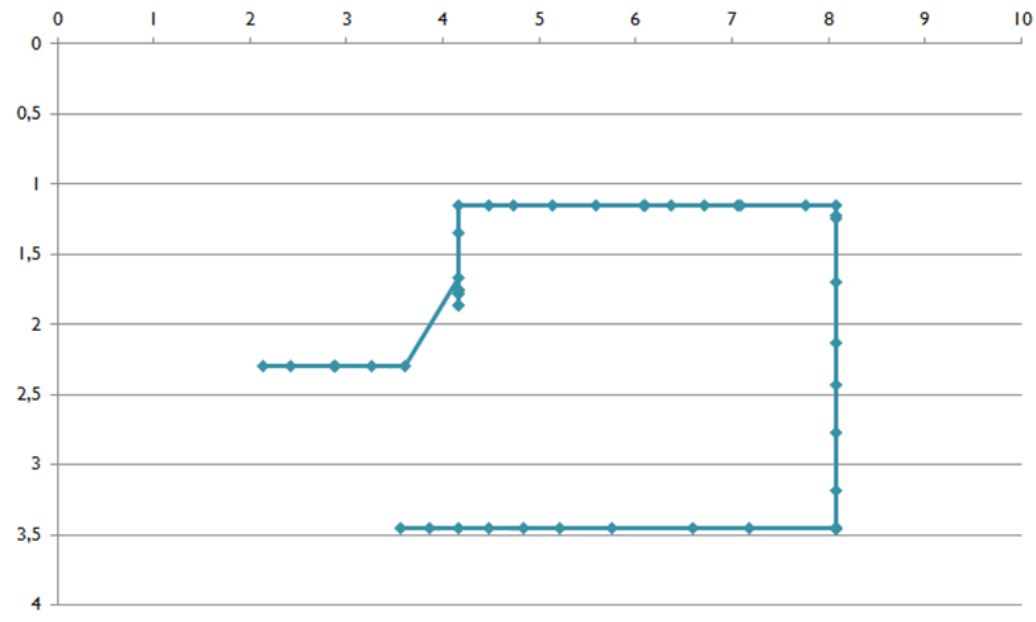

Fig. 8. Total displacement while normal walking

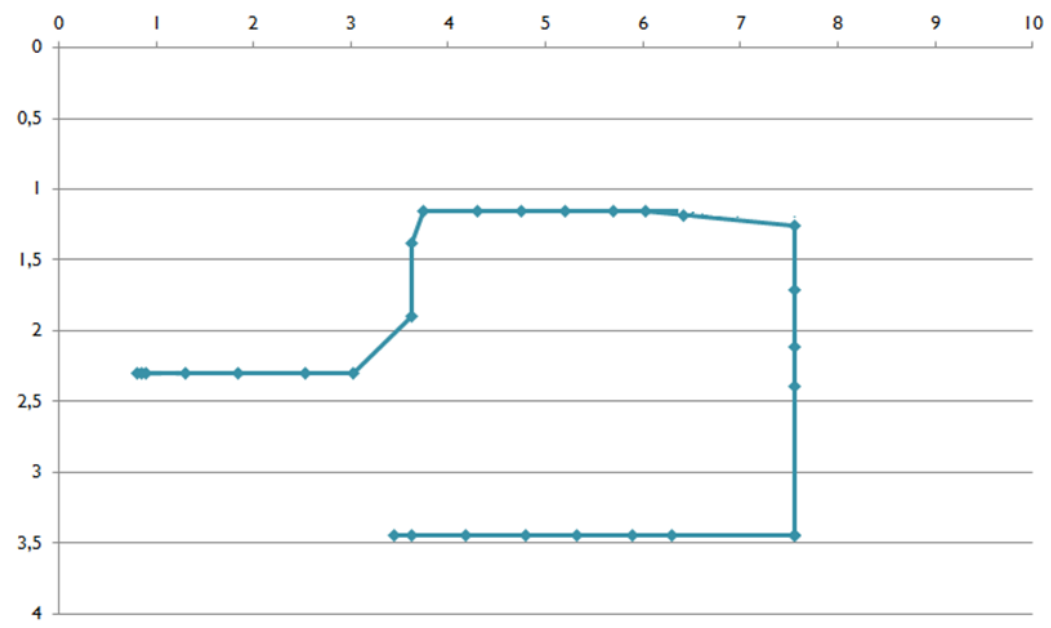

Fig. 9. Total displacement while running

\section{CONCLUSION}

In this paper, we proposed the PDR system for indoor position tracking which is based on fuzzy logic walking status to enhance the accuracy of step length estimation. Proposed system have three main part which are step detection, heading and step length to accomplish sufficient position estimation. Step detection of system has done peak detection of accelerometer values. Heading is estimated by both magnetometer and IMU sensors. Walking status is estimated by FLWS algorithm and positon of pedestrian is estimated. Experimental results show that proposed system provide a good estimation performance.

This study was supported by Kırıkkale University Scientific Research Projects Coordination Unit as BAP project (Project No:2016/127).

\section{REFERANSLAR}

A.R. Jimenez et al., "A Comparison of Pedestrian Dead-Reckoning Algorithms suing a Low-Cost MEMS IMU", IEEE International Symposium on Intelligent Signal Processing, 26-28 August, 2009 Budapest, Hungary

C.-H. Huang, L.-H. Lee, C. C. Ho, L.-L. Wu, and Z.-H. Lai, "Realtime RFID indoor positioning system based on kalman-filter drift removal and heron-bilateration location estimation," Instrumentation and Measurement, IEEE Transactions on, vol. 64, no. 3, pp. 728-739, 2015.

E. Foxlin. Pedestrian tracking with shoe-mounted inertial sensors. IEEE Comput. Graph. Appl., 25(6):38-46, 2005. 
G. Retscher, E. Moser, D. Vredeveld, D. Herberling, "Performance and accuracy test of the WLAN indoor positioning system 'ipo"', Proc. 3rd Workshop on Positioning, Navigation and Communcation, pp. 7-15, 2006

Judd T, Levi R W. Dead reckoning navigational system using accelerometer to measure foot impacts: U.S. Patent 5,583,776[P]. 1996-12-10.

Kim, Jeong Won, et al. "A step, stride and heading determination for the pedestrian navigation system." Positioning 1.08 (2004):0.

K. JongBae and J. HeeSung, "Vision-based location positioning using augmented reality for indoor navigation," IEEE Trans. Consum. Electron., vol. 54, no. 3, pp. 954-962, Aug. 2008

Lauro Ojeda and Johann Borenstein. Non-gps navigation with the personal dead-reckoning system. In SPIE Defense and Security Conference, Unmanned Systems Technology IX, April 9-13 2007.

N. Samama. Global Positioning: Technologies and Performance, volume 1. Wiley-Blackwell, 2008.

Riebeck M. Stark, A. and J. Kawalek. How to design an advanced pedestrian navigation system: Field trial results. In 4th IEEE Workshop on Intelligent Data Acquisition and Advanced Computing Systems: Technology and Applications, IDAACS, April, 15 2007.

S.J. Ingran, D. Harmer, M. Quinlan,"UltraWideBand indoor positioning systems and their use in emergencies", Proc. Position, Location and Navigation Symposium, pp. 706-715,2004.

Widyawan, M. Klepal, and S. Beauregard. A backtracking particle filter for fusing building plans with pdr displacement estimates. In Proc. 5th Workshop on Positioning, Navigation and Communication WPNC 2008, pages 207-212, 27-27 March 2008.

Zadeh, Lotfi A. "Fuzzy sets." Information and control 8.3 (1965): 338-353. 